\title{
Properties of calcium fluoride up to 95 kbar: A theoretical study
}

\author{
CHUN-SHENG WANG \\ School of Traffic and Transportation, Lanzhou Jiaotong University, Lanzhou 730070, PR China
}

MS received 8 July 2008; revised 12 April 2009

\begin{abstract}
We have studied the high-pressure and high-temperature behaviour of calcium fluoride $\left(\mathrm{CaF}_{2}\right) \mathrm{using}$ molecular dynamics simulations with the Born-Mayer-Huggins potentials. The thermal (pressure-volume) equation of state, radial distribution functions (RDFs) just nearby the melting temperature, isothermal bulk modulus, and volume thermal expansion coefficients were calculated from the isobaric and isothermal ensemble. Structural and thermodynamical properties at ambient temperature were well reproduced for the cubic fluorite-type phase of $\mathrm{CaF}_{2}$. At an extended pressure and temperature ranges, the thermal expansivity and bulk modulus of $\mathrm{CaF}_{2}$ have also been predicted. The RDFs indicated that the melting temperature is bracketed between 1600 and $1700 \mathrm{~K}$, and the thermodynamic properties of $\mathrm{CaF}_{2}$ with fluorite-type cubic structure are investigated in the pressure range $0-95 \mathrm{kbar}$ and temperature up to $1600 \mathrm{~K}$.
\end{abstract}

Keywords. Thermodynamic properties; thermal expansion; bulk modulus; calcium fluoride.

\section{Introduction}

Considering the high technological importance of calcium fluoride $\left(\mathrm{CaF}_{2}\right)$, it is not surprising, that during the last years, it has been the subject of many experimental and theoretical studies (Gan et al 1992; de Leeuw and Cooper 2003; Verstraete and Gonze 2003). The superionic conductor, $\mathrm{CaF}_{2}$, crystallize in the cubic fluorite structure and constitute an important class of relatively simple ionic crystals whose optical and lattice-dynamical properties are of much theoretical and experimental interest (Samara 1976; Puchina et al 1998; Schmalzl et al 2003). Besides the technological importance of such fluoritetype crystals as superionic conductors, $\mathrm{CaF}_{2}$ has been proposed in the earlier $80 \mathrm{~s}$ as an internal pressure standard in single-crystal diffraction experiments covering simultaneously high-temperature and high-pressure conditions (Hazen and Finger 1981). On the other hand, $\mathrm{CaF}_{2}$ is a well known mineral and the properties at elevated temperature and pressure are of interest to the geophysicist (Kanchana et al 2003).

New computational techniques have led to calculations of the structural phase stability and thermodynamic properties of $\mathrm{CaF}_{2}$. Kanchana and co-workers investigated the structural and electronic properties of $\mathrm{CaF}_{2}$ by using tight binding linear muffin tin orbital method (Kanchana et al 2003). They found that $\mathrm{CaF}_{2}$ undergoes a structural phase transition to the orthorhombic structure at a pressure of around $91 \mathrm{kbar}$. First principle pair-wise simulations and quantum-mechanical $a b$ initio perturbed ion

(wangcs_lzjtu@yeah.net) calculations have been extensively carried out to obtain the static equation of state of the cubic fluorite-type structure and orthorhombic $\mathrm{PbCl}_{2}$-type structure of $\mathrm{CaF}_{2}$ by Pendas et al (1994). The recent high-pressure and high-temperature equation of state for cubic $\mathrm{CaF}_{2}$ has been reported by Angel (1993), who combined room temperature single-crystal X-ray diffraction (XRD) measurements up to $9 \mathrm{GPa}$ with available thermodynamic data. Angle has remarked on the necessity of a reevaluation of this equation of state due to "disagreements in the literature regarding the value of the bulk modulus of fluorite'. An extensive investigation of the $\mathrm{CaF}_{2}$ response to pressure has been carried out by Gerward et al (1992). They performed synchrotron-radiation XRD studies covering the range $0-450 \mathrm{kbar}$ and observed a phase transition from the cubic to the orthorhombic structure at about $95 \mathrm{kbar}$. The transition was found to be atleast partially quenchable and, according to Angel et al (1992), the kinetics could, therefore, play a decisive role since this kind of transition 'includes thermally activated processes such as atomic ordering within a crystal structure'. The metastable high-pressure phase has also been studied at zero pressure by Gerward et al (1992). However, due to the damage suffered by the single crystal in the phase transition, only partial characterization of the crystal parameters is reported for the orthorhombic polymorph. All in all, these studies have focused mainly to investigate the equilibrium transition pressure and not to explain the mechanism of melting and the thermodynamic behaviour at high pressure and high temperature.

The investigations on high-temperature and highpressure behaviour of solids demonstrate that the thermodynamic parameters such as the volume thermal expan- 
sion coefficient and isothermal bulk modulus are important quantities in the earth and material science as they often occur in equations which describe many important properties of materials. Meanwhile, thermal expansion has been related to other thermodynamic parameters through Grüneisen rules. It is a factor in the equations describing many important properties of solids, and it together with specific heat is essential for predicting a thermodynamic equation of state. In order to understand the nature of $\mathrm{CaF}_{2}$ at high pressures it is necessary to use some form of microscopic modeling or simulation. The aim of the present study is to perform molecular dynamics simulations of $\mathrm{CaF}_{2}$, using the empirical pair-wise potential that reproduce structure and thermodynamic data at high pressure and high temperature. The advantages of the computer simulations based on interatomic interactions lies in demonstrating the mechanisms of atomistic processes, and extending this capacity to evaluate material properties to regimes where direct laboratory measurements are difficult or impossible to perform.

In the following section, we briefly describe the interatomic potential and calculated method used in this work, and in $\S 3$, we present and discuss the radial distribution functions (RDFs) just nearby the melting temperature using molecular dynamics method with effective pairwise interactions, and to predict the thermodynamic properties of $\mathrm{CaF}_{2}$ at extreme conditions (up to $95 \mathrm{kbar}$ and $1600 \mathrm{~K}$ ) where reliable experimental data are scarce. Meanwhile, structure and thermodynamic parameters including the lattice parameter, volume thermal expansion coefficient and isothermal bulk modulus are calculated. The paper ends with the main conclusions of our investigation in $\$ 4$.

\section{Computational technique}

The interatomic potential models of molecular dynamics simulation for the alkaline-earth fluorides system have been proposed by Hirao and Kawamura (1994), Belashchenko (1997) and many other researchers. These interatomic potential models show good agreement with structural properties of solid, glass and liquid phases measured by experiments. In this study, the potential energy for molecular dynamics simulation was calculated by the summation of pair-wise interactions between ions $i$ and $j$ that was the Born-Mayer-Huggins (BMH) form of (1):

$$
V_{i j}\left(r_{i j}\right)=Z_{i} Z_{j} e^{2} / r_{i j}+A_{i j} \exp \left(-r_{i j} / \rho_{i j}\right)-C_{i j} r_{i j}^{-6},
$$

where $r_{i j}$ is the interatomic distance between ions $i$ and $j$, $Z_{i}$ or $Z_{j}$ is an effective charge, $e$ the electronic unit charge, $A_{i j}$ and $\rho_{i j}$ the repulsion parameters for ionic cores of pair, $C_{i j}$ the van der Waals constant and $C_{i j} r_{i j}^{-6}$ describe effective dipole-dipole interaction. The $\mathrm{BMH}$ interatomic potential parameters for $\mathrm{CaF}_{2}$ were $A_{--}=1808 \mathrm{eV}$, $\rho_{--}=0.239 \AA, C_{--}=109.1 \mathrm{eV} \AA^{6} ; A_{+-}=679.3 \mathrm{eV}, \rho_{+-}=$ $0.336 \AA, C_{+-}=A_{++}=C_{++}=0$ (Gillan 1986). In order to verify a reliable performance of the potential model used in this simulation, we performed relevant molecular dynamics simulations. Figure 1 shows a comparison of our calculated pressure and volume relation and the experimental data were obtained by combining synchrotron-radiation XRD techniques for $\mathrm{CaF}_{2}$ (Gerward et al 1992). $V_{0}$ is volume at ambient temperature and pressure. The simulated $300 \mathrm{~K}$ isotherms are in good agreement with experimental data up to the pressure of more than 950 kbar. Therefore, we conclude that our interatomic potential works well.

The molecular dynamics simulations were carried out using the isobaric and isothermal ensemble. Temperature is controlled by velocity scaling method. Pressure is controlled by Parrinello and Rahmann method at atmospheric pressure. The $\mathrm{CaF}_{2}$ crystal structure was composed of 324 (Ca 108 and $\mathrm{O} 216$ ) atoms according to an array of $3 \times 3 \times 3$ unit cells of fluorite structure. All simulations have been verified using systems of 324 atoms and we have not noticed relevant differences. The periodic boundary conditions were employed for the simulation system. To avoid excessive computing time, the long-range Coulomb potential was approximated using only the real-space part of the Ewald sum as given by Montani (1992). The equations of motion were integrated by fifth-order Gear's predictor-corrector algorithms using a time step, $\Delta t=0.5 \mathrm{fs}$. It was found that correct results could normally be obtained with the number of time steps, $n=5000$, for equilibration and measurement, respectively. Still, whenever we suspected that the results might have been affected by the choice of the above parameters, we varied them to verify that the final results are correct.

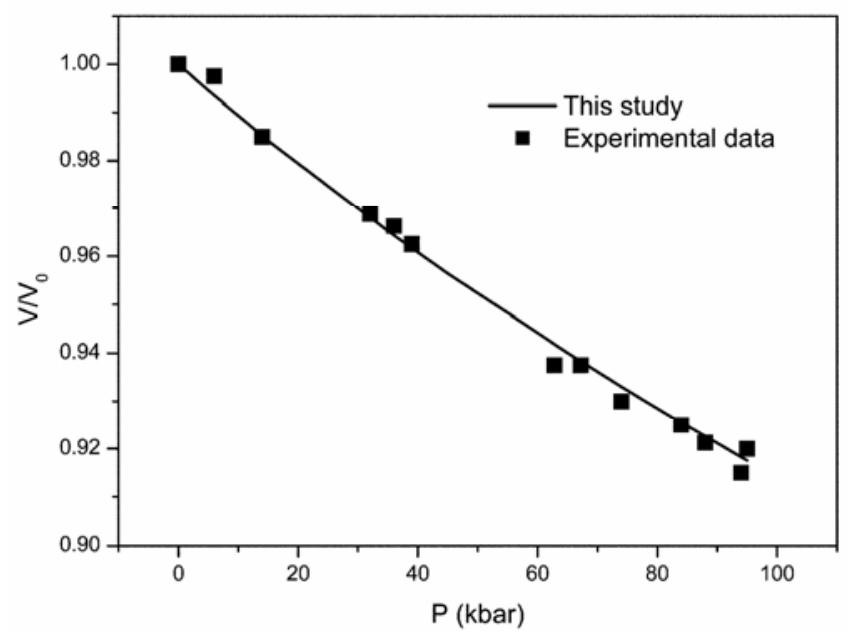

Figure 1. The molecular dynamics calculated isotherms of $\mathrm{CaF}_{2}$ at $300 \mathrm{~K}$, in comparison with the XRD experimental data (Gerward et al 1992). Solid line is the molecular dynamics calculated values for this study. 
Table 1. Structural data for the unit cells of the cubic fluorite-type and orthorhombic $\mathrm{PbCl}_{2}$-type phases of $\mathrm{CaF}_{2}$.

\begin{tabular}{lll}
\hline Crystallographic system & \multicolumn{1}{c}{ Cubic fluorite-type phase } & Orthorhombic $\mathrm{PbCl}_{2}$-type phase \\
\hline Space group & $\begin{array}{l}\mathrm{Fm} 3 m \\
\begin{array}{l}a=b=c \\
\alpha=\beta=\gamma=90\end{array}\end{array}$ & $\begin{array}{l}\text { Pbnm } \\
a \neq b \neq c \\
\alpha=\beta=\gamma=90\end{array}$ \\
Atomic positions & $\mathrm{Ca}(4 a)(0,0,0)$ & $\mathrm{Ca}(4 c)\left(x(\mathrm{Ca}), y(\mathrm{Ca}), \frac{1}{4}\right)$ \\
& $\mathrm{F}(8 c)\left(\frac{1}{4}, \frac{1}{4}, \frac{1}{4}\right)$ & $\mathrm{F} 1(4 c)\left(x(\mathrm{~F} 1), y(\mathrm{~F} 1), \frac{1}{4}\right)$ \\
& & $\mathrm{F} 2(4 c)\left(x(\mathrm{~F} 2), y(\mathrm{~F} 2), \frac{1}{4}\right)$
\end{tabular}

Table 2. Summary of the structure and thermodynamic parameters for the fluorite-type phase of $\mathrm{CaF}_{2}$. Subscript 0 refers to a parameter evaluated at null compression.

\begin{tabular}{lclc}
\hline & This study & \multicolumn{1}{c}{ Calculated } & \multicolumn{1}{c}{ Experimental } \\
\hline$a_{0,300}(\AA)$ & $5 \cdot 375$ & $5 \cdot 173^{[\mathrm{a}]}, 5 \cdot 333^{[\mathrm{a}]}, 5 \cdot 340^{[\mathrm{b}]}, 5 \cdot 520^{[\mathrm{b}]}$ & $5 \cdot 463^{[\mathrm{c}]}, 5 \cdot 460^{[\mathrm{d}]}, 5 \cdot 444^{[\mathrm{e}]}$ \\
$B_{0,300}(\mathrm{kbar})$ & $922 \cdot 5$ & $1030 \cdot 0^{[\mathrm{f}]}, 847 \cdot 0^{[\mathrm{g}]}, 827 \cdot 0^{[\mathrm{g}]}, 930 \cdot 0^{[\mathrm{b}]}$ & $810 \cdot 0^{[\mathrm{h}]}, 827 \cdot 1^{\mathrm{dd}]}, 817 \cdot 0^{[\mathrm{i}]}$ \\
$\alpha_{0,300}\left(10^{-5} \mathrm{~K}^{-1}\right)$ & $4 \cdot 479$ & $5 \cdot 770^{[\mathrm{j}]}$ & \\
\hline
\end{tabular}

\footnotetext{
${ }^{[\mathrm{a}]}$ Schmalzl et al (2003); ${ }^{[\mathrm{b}]}$ Shi et al (2005); ${ }^{[\mathrm{c}]}$ Hazen and Finger (1981); ${ }^{[\mathrm{d}]}$ Nicolav (2000); ${ }^{[\mathrm{e}]}$ White (1980); ${ }^{[\mathrm{f}]}$ Kanchana et al (2003); ${ }^{\mathrm{g}]}$ Pendas et al (1994); ${ }^{\mathrm{h}]}$ Angel (1993); ${ }^{[\mathrm{i}]}$ Wong and Schuele (1968); ${ }^{\left[{ }^{[j]}\right.}$ Schumann and Neumanm (1984).
}

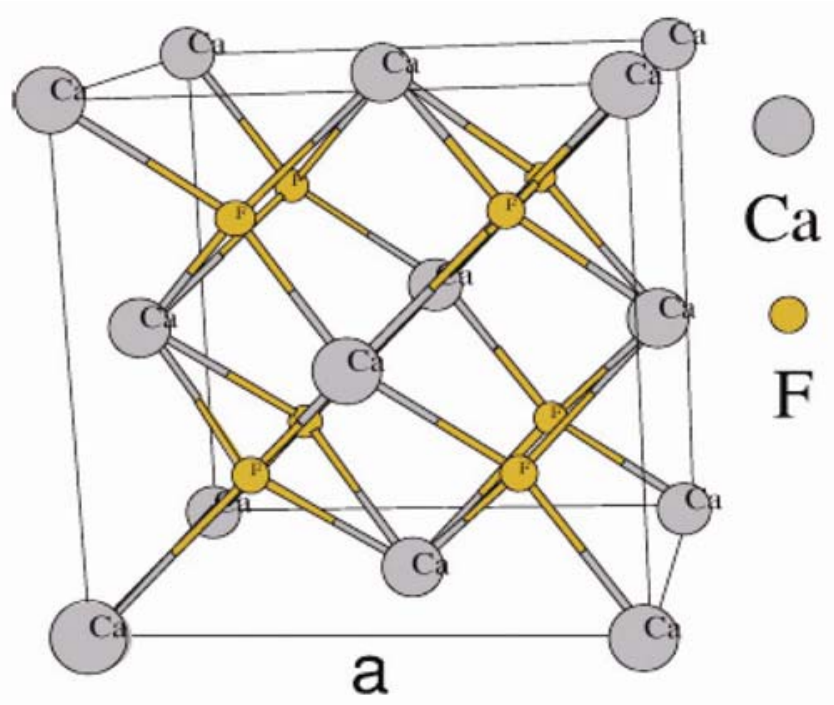

Figure 2. Crystal structure of $\mathrm{CaF}_{2}$. The cube contains four formula units; a complete octahedron is spanned by the $\mathrm{Ca}^{2+}$ ions on the face centres of the cube, while a tetrahedron is spanned by a $\mathrm{Ca}^{2+}$ ion on a cube corner and the three $\mathrm{Ca}^{2+}$ ions on the adjacent face centres.

\section{Results and discussions}

The actual crystalline phase exhibited by $\mathrm{CaF}_{2}$ depends experimentally on the history of the sample as well as on the pressure and temperature conditions. The unit cell descriptions of the cubic fluorite-type and orthorhombic $\mathrm{PbCl}_{2}$-type structures of this compound are collected in table 1 . The cubic fluorite-type structure belongs to the Fm $3 m$ space group, being the cubic cell length $(a)$, its single free geometrical parameter. The orthorhombic $\mathrm{PbCl}_{2}$-type structure belongs to the orthorhombic Pbnm space group and is characterized by specifying nine values: the three unit cell lengths $(a, b, c)$ and the $x$ and $y$ coordinates of its three non-equivalent ions, one calcium (Ca) and two fluorines (F1, F2). For the cubic fluoritetype structure, it is well-known that $\mathrm{CaF}_{2}$ crystallizes in the $f c c$ structure with three atoms in the unit cell. The crystal volume can be viewed as being composed of tetrahedra and octahedra with the $\mathrm{Ca}^{2+}$ ions at the corners and the $F^{-}$ions at the centres of the tetrahedra (see figure 2). In the present work, a critical comparison between experimental and theoretical results is possible for $\mathrm{CaF}_{2}$ as the structural and compressive parameters can be measured with a fair level of accuracy since the solid adopts simple structure: cubic fluorite-type structure.

The radial distribution function (RDF) is one of the most important structural quantities characterizing a system, particularly for liquids. Melting and crystallizing can also be identified from the long-range disorder and order manifested in RDFs. Figure $3(\mathrm{a}-\mathrm{c})$ shows the structure of the cubic fluorite-type phase of $\mathrm{CaF}_{2}$ at zero pressure, and at 1600 and $1700 \mathrm{~K}$, just nearby the real melting temperature. Zen et al (2008) found the melting temperature of 

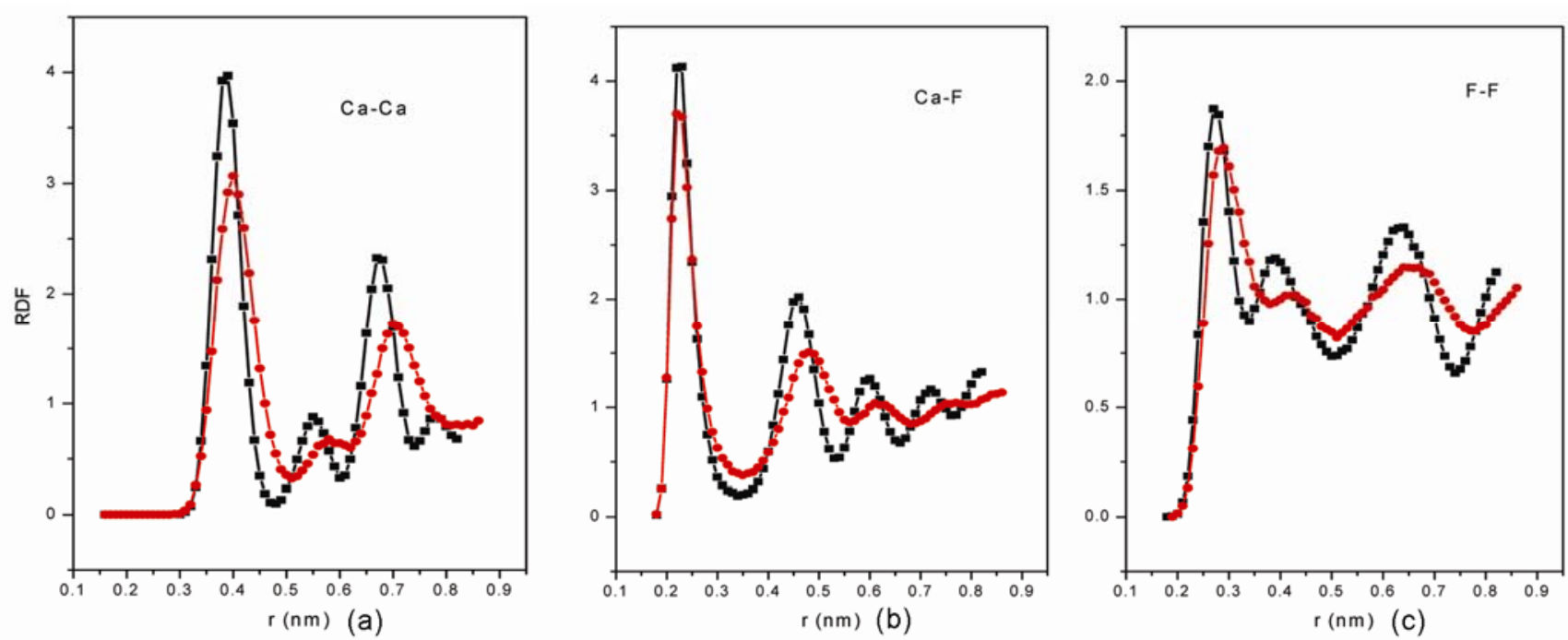

Figure 3. Radial distribution functions of the cubic fluorite-type phase of $\mathrm{CaF}_{2}(\mathbf{a}-\mathbf{c})$ at zero pressure. The closed squares and the closed circles are the molecular dynamics calculation values at temperatures, 1600 and $1700 \mathrm{~K}$, just nearby the melting temperature, $1650-1733 \mathrm{~K}$, at room pressure (Zeng et al 2008), respectively.

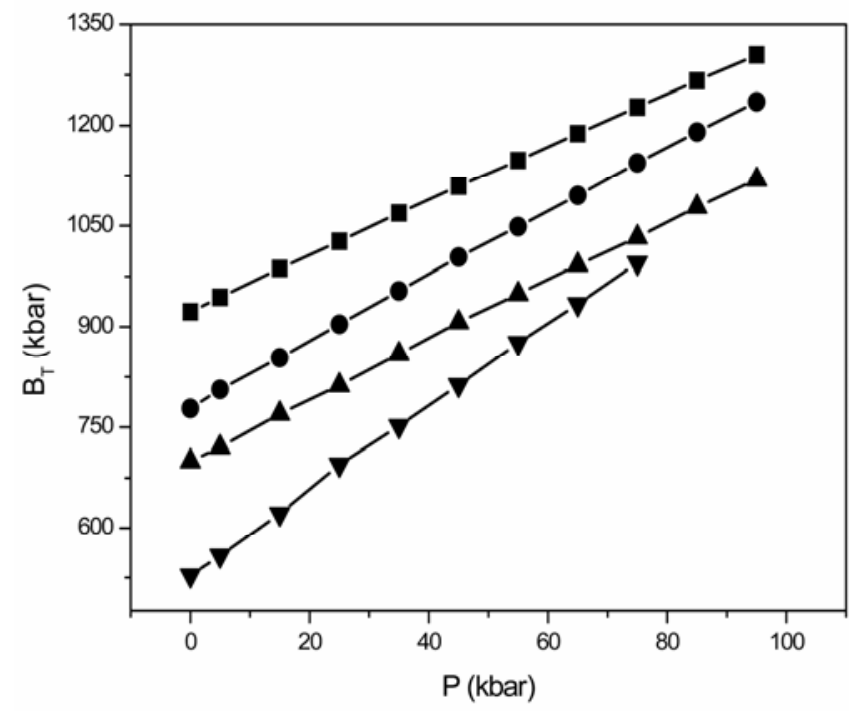

Figure 4. Isothermal bulk modulus of $\mathrm{CaF}_{2}$ with cubic fluorite-type structure versus pressure. The closed squares, the closed circles, the closed up triangles, and the closed down triangles are the molecular dynamics calculation values at 300 , 800,1200 , and $1600 \mathrm{~K}$, respectively.

$\mathrm{CaF}_{2}$ is about $1650-1733 \mathrm{~K}$ at room pressure, which is very close to the present findings.

The RDFs for the simulated system can be calculated by (2):

$$
g_{i j}(r)=\frac{V}{N_{i} N_{j}} \sum_{j} \frac{\left\langle n_{i j}(r-\Delta r / 2, r+\Delta r / 2)\right\rangle}{4 \pi r^{2} \Delta r},
$$

where $\left\langle n_{i j}(r-\Delta r / 2, r+\Delta r / 2)\right\rangle$ is the average number of ion, $j$, surrounding ion, $i$, in a spherical shell within $r \pm \Delta r / 2 . N_{i}$ and $N_{j}$ are the total number of ions $i$ and $j, V$ the volume of the system. Note the changes of cubic fluorite-type structure of $\mathrm{CaF}_{2}$ from a solid to a liquid when they are heated. As one can see, the difference among two structures is quite evident. The RDFs of $\mathrm{Ca}-\mathrm{Ca}$, $\mathrm{Ca}-\mathrm{F}$, and $\mathrm{F}-\mathrm{F}$ ion pairs rather feature solid structure at $1600 \mathrm{~K}$. When the temperature is $1700 \mathrm{~K}$, the RDFs of these ion pairs show an obvious first peak, a visible second peak and a featureless tail. We want to emphasize that the absence of strong peaks in the RDFs at distances larger than the position for the second peak is typical for non-crystalline structure and the fluorite-type structure loses its long-range order and begins to melt. So, melting temperature is bracketed between 1600 and $1700 \mathrm{~K}$. In this paper the thermodynamic properties of $\mathrm{CaF}_{2}$ with fluorite-type cubic structure are investigated in the pressure range 0-95 kbar and temperature up to $1600 \mathrm{~K}$.

For the cubic fluorite-type structure of $\mathrm{CaF}_{2}$ there is a number of zero-pressure observable properties which can be obtained with various experimental and computational methods. Table 2 shows the comparison of the structure and thermodynamic parameters including lattice parameter, volume thermal expansion coefficient, and isothermal bulk modulus of $\mathrm{CaF}_{2}$ at ambient condition with experiments and other calculations in detail. We calculated lattice parameter for the fluorite-type structure of $\mathrm{CaF}_{2}$ and the value is $5.173 \AA$, which is in good agreement with the theoretical and experimental values (White 1980; Hazen and Finger 1981; Nicolav 2000; Schmalzl et al 2003; Shi et al 2005). The isothermal bulk modulus is defined as the volume derivative of pressure, $B_{T}=-V(\partial P / \partial V)_{T}$. XRD leads to the determination of the isothermal bulk modulus. Besides the experimental investigations, many theoretical calculations have also been employed to deter- 
mine the structural and mechanical properties of $\mathrm{CaF}_{2}$. The value of the bulk modulus for $\mathrm{CaF}_{2}$ in the present work is $922.5 \mathrm{kbar}$. The isothermal bulk modulus is also accessible from the fitting of empirical equations to pressure-volume data. Thus the Murnaghan equation of state derived from room temperature measurements of Angel (1993) produces a value of 810 for $B_{0}$, which agrees with room temperature isothermal value of $817 \mathrm{kbar}$ obtained from the ultrasonic measurements of Wong and Schuele (1968). Therefore, Angel's results would suggest that the athermal value for $B_{0}$ were closer to $900 \mathrm{kbar}$, as in the ultrasonic experiments, than to $850 \mathrm{kbar}$ as in the Brillouin scattering measurements. We believe, however, that both extrapolated values are not sufficiently different with each other to refuse one in favour of the other.

In the context of the bulk modulus, it is also of interest to investigate the isothermal bulk modulus, $B_{T}$, under various pressures and at various temperatures. In figure 4 , we illustrate $B_{T}$ as a function of pressure up to $95 \mathrm{kbar}$ at temperatures of $300,800,1200$ and $1600 \mathrm{~K}$. It is obvious that the isothermal bulk modulus of $\mathrm{CaF}_{2}$ with cubic fluorite-type structure increases with increasing pressure, and decreases with increasing temperature.

It has been emphasized that most of the large errors in the calculations of thermodynamic functions arise due to the uncertainty of thermal expansivity. We think that application of molecular dynamics tools for the calculation of thermal expansivity properties is a viable strategy for obtaining some of the available information. The temperature dependence of the volume, and the thermal expansion coefficients of $\mathrm{CaF}_{2}$ defined as $\alpha_{P}=(1 / V)$ $(\partial V / \partial T)_{P}$ has been determined here. The volume thermal expansion coefficient at $300 \mathrm{~K}$ for the cubic fluorite-type

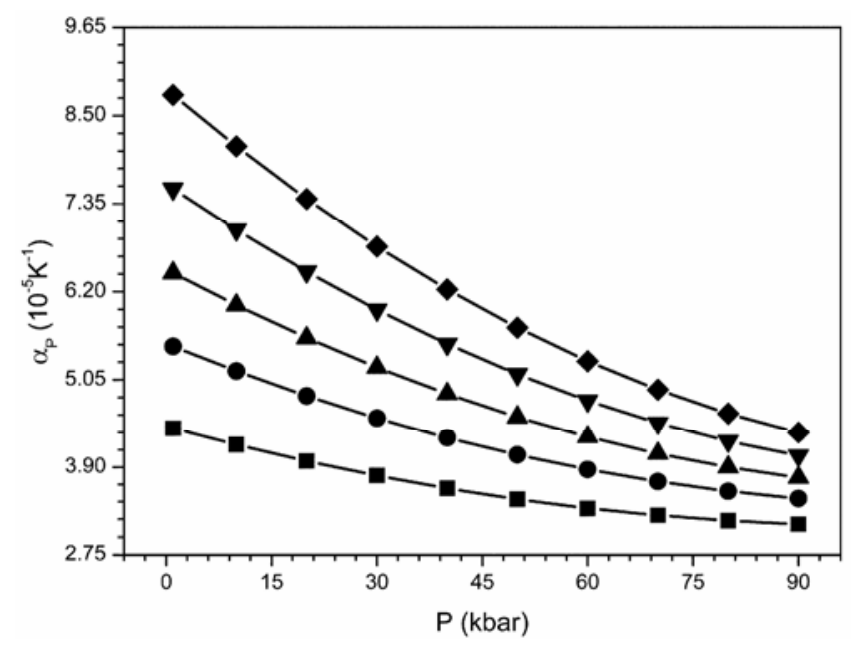

Figure 5. The volume thermal expansion coefficients of $\mathrm{CaF}_{2}$ with cubic fluorite-type structure versus pressure. The closed squares, the closed circles, the closed up triangles, the closed down triangles, and the closed diamonds are the molecular dynamics calculation values at 300, 700, 1000, 1300, and $1600 \mathrm{~K}$, respectively. structure of $\mathrm{CaF}_{2}$ is given in table 2, and the molecular dynamics simulation is $4.415 \times 10^{-5} \mathrm{~K}^{-1}$. Schumann and Neumanm (1984) gave a value of $5.770 \times 10^{-5} \mathrm{~K}^{-1}$ and good agreement was obtained.

In order to study further the influence of pressure and temperature on thermal expansivity for $\mathrm{CaF}_{2}$, the simulations in an extended pressure and temperature range were performed and shown in figure 5. The thermal expansivity of $\mathrm{CaF}_{2}$ with cubic fluorite-type structure is increased with increasing temperature and the influence of temperature on thermal expansivity at high pressures is small. With the increasing pressure, the volume of the solid is decreased and the atoms come closer to each other, increasing the depth of the potential energy well and reducing the anharmonic nature of the potential energy curve at high temperature. Since, thermal expansivity is a result of anharmonicity in the potential energy, it becomes virtually independent of pressure and temperature in the high-pressure and -temperature domains. The knowledge of thermal expansivity can help to constrain other important parameters such as Grüneisen parameters and Rayleigh number. For the thermal expansivity, experimental measurements and other theoretical calculations are needed in order to confirm our results and also to know more about the thermodynamic properties of $\mathrm{CaF}_{2}$ under pressure.

\section{Conclusions}

We have calculated a number of structural and thermodynamical properties including pressure-volume equation of state, lattice parameter, isothermal bulk modulus and thermal expansion coefficients of $\mathrm{CaF}_{2}$ with cubic fluorite-type structure using molecular dynamics simulations with the Born-Mayer-Huggins potentials. Our calculated thermodynamic parameters are in good agreement with well-established experimental data and existing theoretical results. Isothermal bulk modulus and thermal expansion coefficients of $\mathrm{CaF}_{2}$ with cubic fluorite-type structure have made reliable predictions. It can be found that the isothermal bulk modulus of $\mathrm{CaF}_{2}$ decreases with increasing temperature and increases with increasing pressure. The thermal expansivity of $\mathrm{CaF}_{2}$ at low pressures range is increased with increasing temperature. On the other hand, high-pressure and high-temperature thermodynamic properties of $\mathrm{CaF}_{2}$ determined here can now be used for modeling the composition in the earth's lower mantle.

\section{References}

Angel R J 1993 J. Phys.: Condens. Matter 5141

Angel R J, Ross N L, Wood I G and Woods P A 1992 Phase Trans. 3913

Belashchenko D K 1997 Russ. Chem. Rev. 66733

de Leeuw N H and Cooper T G 2003 J. Mater. Chem. 1393 
Gan F, Xu Y N, Huang M Z, Ching W Y and Harrison J G 1992 Phys. Rev. B45 8248

Gerward L, Olsen J S, Steenstrup S, Malinowski M, Asbrink S and Waskowska A 1992 J. Appl. Cryst. 25578

Gillan M J 1986 J. Phys. C19 3391

Hazen R M and Finger L W 1981 J. Appl. Cryst. 14234

Hirao K and Kawamura K 1994 Material design using personal computer (Tokyo: Shokabo) p. 52

Kanchana V, Vaitheeswaran G and Rajagopalan M 2003 Physica B328 283

Montani R A 1992 J. Phys. Chem. Solids 531211

Nicolav M 2000 J. Cryst. Growth 21862

Pendas A M, Recio J M, Florez M, Luana V and Bermejo M 1994 Phys. Rev. B49 5858
Puchina A V, Puchin V E, Kotomin E A and Reichling M 1998 Solid State Commun. 106285

Samara G A 1976 Phys. Rev. B13 4529

Schmalzl K, Strauch D and Schober H 2003 Phys. Rev. B68 144301

Schumann B and Neumanm H 1984 Cryst. Res. \& Technol. 19 13

Shi H, Eglitis R I and Borstel G 2005 Phys. Rev. B72 045109

Verstraete M and Gonze X 2003 Phys. Rev. B68 195123

White G K 1980 J. Phys. C13 4905

Wong C and Schuele D E 1968 J. Phys. Chem. Phys. 29 1309

Zen Z Y, Chen X R, Zhu J and Gu C E 2008 Chin. Phys. Lett. 25230 